\title{
Surgical approaches to paraspinal nerve sheath tumors
}

\author{
Alice Cherqui, M.D., ${ }^{1}$ DAniel H. Kim, M.D. ${ }^{2}$ Se-Hoon Kim, M.D., Ph.D., ${ }^{2,3}$ \\ Hyung-Ki Park, M.D., ${ }^{4}$ AND DaVID G. KLINE, M.D. ${ }^{1}$ \\ ${ }^{1}$ Department of Neurosurgery, Louisiana State University Health Sciences Center; \\ ${ }^{2}$ Department of Neurosurgery, Ochsner Clinic Foundation, New Orleans, Louisiana; \\ ${ }^{3}$ Department of Neurosurgery, Korea University Medical Center; and ${ }^{4}$ Department of \\ Neurosurgery, Soonchunhyang University Hospital, Seoul, Korea
}

\begin{abstract}
Object. The goal of this study was to analyze the results of surgical treatment of paraspinal nerve sheath tumors (NSTs) and review the surgical approaches to paraspinal NSTs.

Methods. A retrospective review of the cases of paraspinal NSTs treated surgically by two senior authors during the period between 1970 and 2006 was undertaken. Surgical approaches that allow minimal disruption of normal anatomy and are aimed at complete resection of paraspinal lesions and preservation of spinal stability are reviewed according to the spinal level.

Results. Eighty-eight paraspinal NSTs were treated surgically during the period: 56 schwannomas, seven solitary neurofibromas, 21 neurofibromas associated with neurofibromatosis Type 1 (NF1), and four malignant peripheral NSTs. Schwannomas tended to occur in the cervical and thoracic areas. Neurofibromas were usually associated with NF1 and tended to occur in the cervical area. Pain (79 patients, 90\%) and paresthesia (81 patients, 92\%) were the predominant clinical presenting symptoms; others included weakness (28 patients) and myelopathy (12 patients). Total resection of the tumor was achieved in 50 patients $(89.3 \%)$ with schwannomas and 22 patients $(78.6 \%)$ with neurofibromas. There was a large reduction of pain in $70(88.6 \%)$ of 79 patients who had preoperative pain, and weakness improved in $18(64.3 \%)$ of 28 . Postoperative transient weakness occurred in $12(42.9 \%)$ of these patients, but in $85 \%$ of this group, the symptom improved over a 12-month period. Myelopathy was reduced in eight $(66.7 \%)$ of 12 patients. The average follow-up period was 18 months.

Conclusions. Paraspinal NSTs present unique surgical challenges given their anatomical relationships to the spine, spinal cord, nerve roots, and major vasculature. The surgical technique should take into account the location of the lesion and its relationship to paraspinal anatomy, the extent of resection, sparing of normal anatomy, and spinal instability.
\end{abstract}

KEY WORDS - paraspinal nerve sheath tumor • retrospective review $\quad$ surgical approach

$\mathrm{P}$ ARASPINAL NSTs are tumors with contiguous extraand intraforaminal components that may extend intraspinally. Their incidence varies with anatomical location. Generally, approximately 10 to $15 \%$ of spinal NSTs have a characteristic dumbbell shape. Paraspinal NSTs are found most often in the cervical spine, followed by the thoracic and lumbar spine. In the cervical area, paraspinal NSTs account for approximately $10 \%$ of all cervical NSTs. Paraspinal NSTs of the thoracic area generally account for $10 \%$ of posterior mediastinal lesions. Lumbosacral paraspinal NSTs are not as well reported but are found less frequently than cervical or thoracic lesions. Schwannomas account for the majority of these lesions, except in NF1, in which neurofibromas predominate.

Abbreviations used in this paper: $\mathrm{CT}=$ computed tomography; $\mathrm{MR}=$ magnetic resonance; NF1 = neurofibromatosis Type 1 ; $\mathrm{NF} 2$ = neurofibromatosis Type 2; NST = nerve sheath tumor; VA $=$ vertebral artery; $\mathrm{VB}=$ vertebral body.
Solitary neurofibromas are less frequent but do occur sporadically. Malignant peripheral NSTs occur in 5\% of patients with plexiform neurofibromas and NF1.

Clinically, patients present with signs and symptoms related to the spinal level of involvement. Pain and paresthesia are the prominent symptoms in 80 to $90 \%$ of patients, but radiculopathy and myelopathy can occur in up to $25 \%$, especially in patients with spinal cord compression and eloquent nerve root involvement. Generally, symptoms improve after surgical resection, although undue delay in treatment can lead to permanent disability.

\section{Clinical Material and Methods}

A retrospective review of all cases of paraspinal NSTs treated surgically by two senior authors (D.H.K. and D.G.K.) during the period between 1970 and 2006 was undertaken. Patients received preoperative and postoperative neurological examinations. Diagnostic studies includ- 
ed myelography and CT in the earlier years of the study period and MR imaging as it became widely used. Some patients also underwent preoperative electromyography. Patients diagnosed with NF1 and NF2 underwent full neuroaxis evaluations. Intraoperative electrophysiological recordings were performed in most of the cases reviewed in this study.

\section{Surgical Considerations Regarding Paraspinal Nerve Sheath Tumors}

Approaches to Cervical and Upper Thoracic Tumors. The level of the paraspinal lesion will determine the surgical approach. Depending on the level, there are different anatomical considerations. Lesions of the cervical spine are best approached through a standard anterior cervical or midline posterior cervical approach and laminectomy (Fig. 1). Instrumented fusion may be required in the treatment of multilevel lesions. A posterior subscapular approach can be added for lesions involving the lower brachial plexus or upper thoracic nerve roots, T-1 through T-3.

Posterior Approach. In a posterior approach, several factors are important. The bony exposure must encompass the tumor and may involve multilevel laminectomies and facetectomies. If needed, spinal stabilization with instrumentation is performed once the tumor has been resected. An occipitocervical fixation is performed for C-1 and C-2 disruption. Lateral mass fixation is used for the lower cervical spine. The VA should be freed. If the VA is embedded in the lesion, the cephalad and caudal portion entering and leaving the tumor mass is exposed for control. The periosteal venous sheath can reveal significant venous bleeding. The veins are best coagulated and stripped from the artery. The VA can be transposed, provided there is complete control of the artery and all of its branches have been secured and sectioned. If the VA is injured during dissection, it may be ligated if there is adequate collateral circulation, or direct repair may be undertaken.

Posterior Subscapular Approach. The posterior subscapular approach to the plexus can be very useful alone or in combination with the posterior cervical approach for the treatment of proximal paraspinal lesions extending intraforaminally and involving the lower brachial plexus (C7-T1) or upper thoracic nerve roots, (T1-T3). Advantages of the posterior subscapular approach include a large exposure and avoidance of an anterior approach to the lower thoracic spine, which is associated with significant morbidity. The major disadvantage of the posterior subscapular approach is that it is not routinely used by most neurosurgeons.

Care should be taken to position the patient. He or she should be placed in the prone position, with the operative side placed close to the edge of the operating table. Rolls are placed under the anterolateral chest wall, transversely beneath both shoulders and the manubrium of the chest. The upper extremity on the operative side is partially abducted and flexed forward at the shoulder. The arm is flexed at the elbow and secured to a padded Mayo stand, which is at a level lower than the operating table. The table is then placed in 15 to $20^{\circ}$ of reverse Trendelenburg to allow further abduction of the shoulder and scapula. The head is then turned away from the operative site and

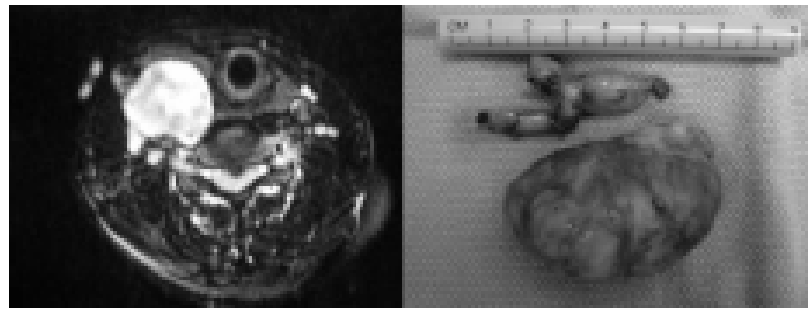

FIG. 1. A case of a cervical paraspinal NST in a 43-year-old woman. Preoperative axial T2-weighted MR image (left) and gross photograph of the tumor (right). The histopathological diagnosis was schwannoma.

appropriately padded to keep pressure off the orbits and maintain an open airway.

The surgical exposure is carried down to the proximal plexus and upper thoracic nerve roots in a careful and deliberate fashion to preserve the normal anatomy. The muscles that connect the spine to the scapula are the trapezius, rhomboid, and levator scapulae. A curvilinear incision is centered between the thoracic spinous processes and medial edge of the scapula. The spinal branch of the accessory nerve and the ascending branch of the transverse cervical artery run near the medial vertebral border of the scapula.

The spinal portion of the trapezius is transected along the length of the incision. It is important to mark the edges with suture for later accurate approximation. The levator scapulae and rhomboids insert on the medial border of the scapula. The levator scapulae lies superiorly, the rhomboid minor medially, and the rhomboid major inferiorly to the trapezius. These muscles are dissected in a segmental fashion and clamped with a Kelly clamp midway between the scapula and spine. They are then sectioned in an inferior to superior direction. The muscle edges are also marked with suture. Dividing the musculature away from the scapula protects the deeper dorsal scapular nerve and ascending branch of the transverse cervical artery. The clamps are removed, and the ends of the sutures are fastened to the drapes. If the dissection approaches the neck, the thicker portion of the trapezius can be split in a medial direction. In addition, some of the serratus posterior muscle may also be divided.

Once the rhomboids are divided, the posterior chest wall can be seen. An avascular plane between the scapula and the shoulder plane and chest wall is created with blunt finger dissection. A chest retractor is placed to expose the area between the scapula mass and the thoracic spinous processes. The retractor is opened as the limb on the padded Mayo stand is lowered or the operating table elevated. This allows maximal abduction and external rotation of the scapula and exposes the posterior aspects of the upper ribs.

The first rib is identified by palpation from the second rib. The scalenus posterior is detached from the superior surface of the first rib. More medial segments of the scalene muscles are resected from their insertion to their origin from the transverse spinous processes. The roots and spinal nerves and trunks are exposed when the muscles are displaced superiorly. The intercostal muscles and soft tissues are stripped from the rib. The first rib is removed ex- 


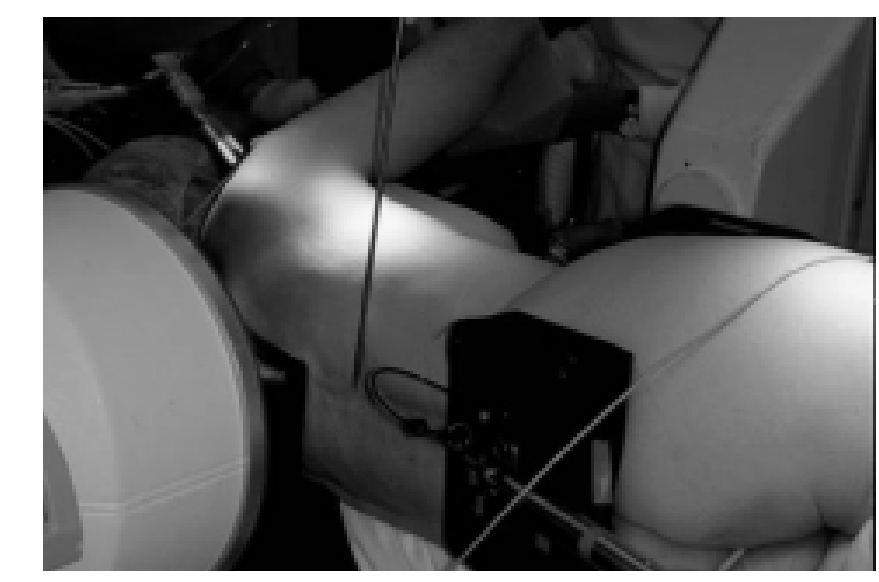

FIG. 2. Photograph showing positioning of the patient and level confirmation in the thoracoscopic approach to a thoracic paraspinal NST. The patient is placed in a stable lateral decubitus position, secured with a four-point support.

traperiosteally to the costotransverse articulation medially and costoclavicular ligament laterally. Care must be taken to avoid entry into the apical pleura as the first rib is removed between the T-1 and C-8 spinal nerves. The rib is resected to a margin 5 to $6 \mathrm{~cm}$ lateral to the junction of the rib and the transverse process. The paraspinous muscles may be elevated and retracted to allow further access to the foraminae, facets, and laminae.

The second rib can also be removed if necessary in very large patients or for the resection of large tumors extending into the mediastinum. A Weitlaner retractor is placed under the second rib and soft tissue of the superior neck to open up the supraclavicular space posterior to the plexus. The lower trunk is isolated and circumferentially dissected from the deeper subclavian artery and vein. The vessels are dissected away from the lower trunk and protected. The middle and upper trunk may also be isolated depending on the location and extent of the tumor. The origin of the long thoracic nerve from the posterior aspect of the C6 and C-7 spinal nerves is seen and can be protected. The phrenic nerve is anterior to the upper trunk and scalenus anterior muscle. For lesions extending intraforaminally, a posterior foraminotomy is performed. The facet joint can also be removed to expose the intraforaminal course of nerve and tumor. The exposure can be expanded further with a facetectomy and laminectomy. Tumor and nerve roots can be unroofed without fear of serious bleeding because the VA is located anterior to the spinal nerves. When the multiple facetectomy is carried out, stabilization should be made with lateral mass screw or pedicle screw fixation systems.

A meticulous closure should be achieved. The anatomical reapproximation of different muscle planes should be respected from previously marked musculature. Any pleural breaches should be repaired. A chest tube is seldom needed.

Many critical and visceral structures are present in the region of the cervicothoracic junction from $\mathrm{C}-7$ to $\mathrm{T}-4$. Paraspinal lesions that may not be fully resected with the combined posterior and posterior subscapular approach may require more advanced approaches to the cervicothoracic junction. The transsternal-transthoracic approach provides exposure down to T-4, especially in obese patients. It offers the best access to T-3 and T-4, but it also is associated with the greatest degree of morbidity of all anterior approaches.

Approaches to Middle and Lower Thoracic Tumors. Lesions of the thoracic and thoracolumbar spine can be approached through a minithoracotomy, a thoracoscopic approach, a costotransversectomy, or a combination of anterior and posterior approaches. Surgical interventions should limit operative time, allow adequate resection, and avoid damage to critical intrathoracic structures.

Identification of the intercostal or lumbar artery from which the artery of Adamkiewicz branches is extremely important in planning surgical treatment. The artery of Adamkiewicz, also known as the great segmental medullary artery, typically arises from a left posterior intercostal artery, which branches from the aorta, and supplies the lower two thirds of the spinal cord via the anterior spinal artery. Anatomical variations are common, however. The artery of Adamkiewicz sometimes arises from a lumbar vessel; in approximately $30 \%$ of people it arises from the right side, and one quarter of people have two great segmental medullary arteries. ${ }^{12}$ Injury to this artery may cause paraplegia.

Thoracoscopic Approach. Neurogenic tumors of the thoracic spine and dumbbell tumors with a large intrathoracic component have been traditionally approached through a conventional open thoracotomy with a combined posterior approach. The morbidity associated with an open thoracotomy limits the application of anterior approaches to the thoracic spine. Access to the anterior column requires rib removal and opening of the parietal pleura, which can cause postthoracotomy syndromes and intercostal neuralgia. Open thoracotomy requires large incisions, and the surgeon's operative view can be obscured by hands or surgical instruments. We have adapted the surgical principles of an open thoracotomy to a less invasive "mini-approach" to the mid and lower thoracic spine. Video-assisted thoracic surgery has been essential in expanding the small microscopic views of minimally invasive open microscopic approaches. Patients with significant cardiopulmonary disease, acute posttraumatic lung failure, or disturbances of hemostasis are not candidates for this approach.

The thoracoscopic approach requires a standard surgical set and the proper endoscopic instruments: videoendoscopy equipment and instruments for thoracoscopic dissection of prevertebral anatomical structures and resection of bone and ligament. The patient is positioned in a lateral decubitus position with standard four-point support (Fig. 2). The upper arm is abducted and elevated to avoid interference with the endoscope. Monitors are placed at the lower end of the operating table on opposite sides to enable free vision for the surgeon and assistants. The $\mathrm{C}$ arm monitor is placed on the opposite side. The borders of the lesion vertebra are identified and marked on the skin. The working channel is centered over the target vertebra. The optical channel is placed between two and three intercostal spaces above the target vertebra for lesions at the thoracolumbar junction. The optical channel is placed below the target vertebra for lesions of the middle and upper thoracic spine. The portal for suction and irrigation is 


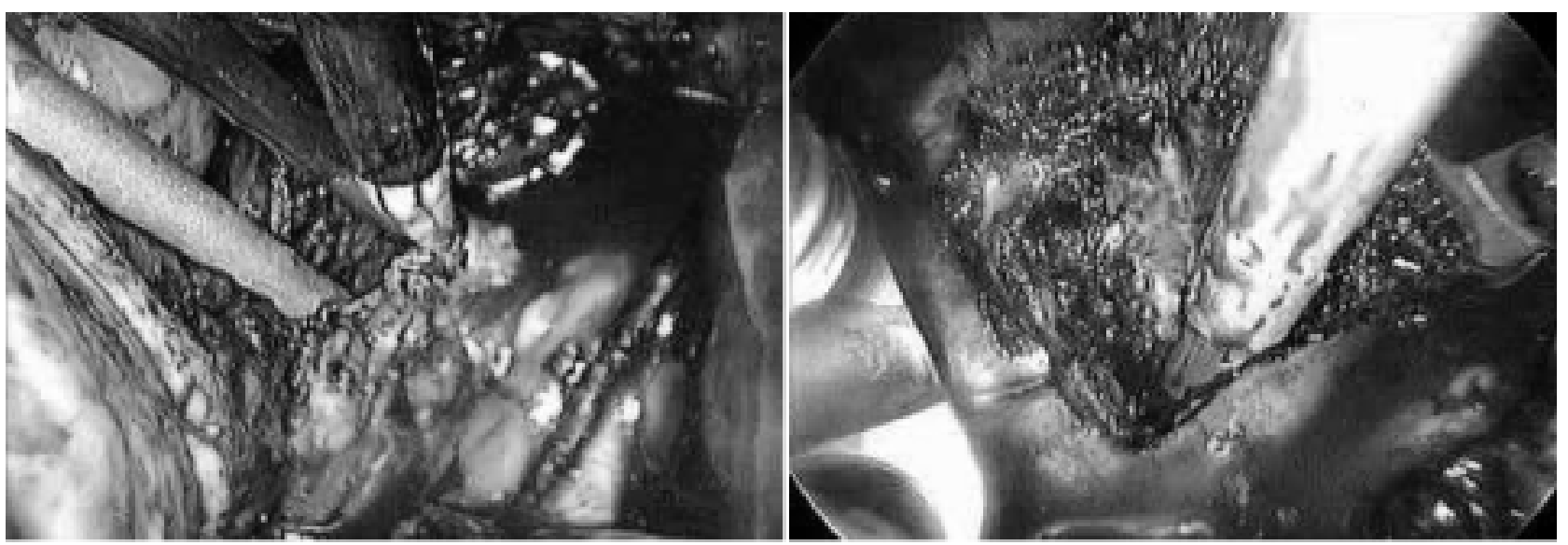

FIG. 3. Intraoperative photographs obtained during a thoracoscopic approach to a paraspinal NST at the T-9 level, showing extension of the parietal pleura dissection (left) and demarcation of the tumor margin (right).

placed 5 to $10 \mathrm{~cm}$ anterior to the working and optical channel. The portal for the lung retractor is placed as far ventrally as possible to avoid interference with other instruments.

The portals are placed through $1.5-\mathrm{cm}$ incisions above the intercostal space. The muscles of the thoracic wall are bluntly crossed and the intercostal space is opened. The pleura is exposed, and an opening into the thoracic cavity is created. A $10-\mathrm{mm}$ trocar is inserted and one-lung ventilation begins. The $30^{\circ}$ endoscope is inserted at a flat angle in the direction of the second trocar. The second, third, and fourth trocar are inserted under direct visual control through the instrument.

The target vertebra can now be exposed. The fan retractor is placed in the anterior port and is used to sweep back the lung tissue and hold down the insertion of the diaphragm to the spine. The lower thoracic spine is exposed after the pulmonary ligament is cut. In low lesions, the diaphragm is retracted caudally to expose the costodiaphragmatic recess. The parietal pleura is incised with a hook diathermy electrode to expose the tumor mass. The pleural incision is made over the center of the tumor mass and is widely extended to expose and mobilize the tumor margins (Fig. 3). In cystic lesions, fluid aspiration should be performed prior to the pleural incision to facilitate en bloc resection. The segmental vessels are located at the middle of the vertebra and should not be injured during dissection. The exposure can be enlarged from the disk to adjacent VBs. Segmental vessels are coagulated and divided. Significant bone removal is not usually required with thoracoscopic resections of paraspinal tumors. The tumor is resected piece by piece. The intraforaminal portion of the tumor is resected once the tumor is debulked. Adjacent vessels and visceral structure are dissected in a blunt and sharp fashion from tumor. Traction is avoided to prevent avulsion of the nerve root, which may tear the dura and cause cerebral spinal fluid leakage. Patients with dumbbell tumors require a combined posterior and thoracoscopic approach. The posterior approach is performed via a standard midline posterior approach and laminectomy. The intraspinal component must be resected and freed from its relation to the spinal cord. Traction on the spinal cord is thus avoided during the thoracoscopic procedure.
Although the adjacent ribs and vertebrae may be eroded with paraspinal lesions, instrumentation is not usually necessary. However, thoracoscopy allows for reconstruction of corpectomy sites with instrumentation.

Posterior Approaches. Several surgical approaches have been designed to remove thoracic lesions. The surgical approach depends on the disease entity, level of the lesion, multiplicity, presence of instability, and the need for reconstruction. The posterior approaches provide less extensive exposure of the VB than the anterior approach. In the case of paraspinal NST, exposure of the anterior portion of the vertebra is usually not necessary, except in large lesions or when there is large bony involvement. The involved nerve, the lateral aspect of the vertebra, the neural foramina, and the lateral aspect of the spinal canal should be exposed. Posterior and posterolateral approaches allow for a more lateral trajectory to visualize the affected vertebrae better. Depending on the extent of the paraspinal lesion, adjacent vertebral levels may need to be exposed. Posterior approaches offer several advantages. The anatomy is more familiar to neurosurgeons, and the spine may be stabilized with posterior instrumentation devices in one procedure. A thoracotomy is also avoided, which is especially important in patients with anesthetic risk.

Costotransversectomy. The costotransversectomy was first used for drainage of tuberculous paraspinal abscesses in Pott disease. ${ }^{1}$ The approach can be used for patients with thoracic paraspinal lesions, as it allows access to the posterior and lateral aspects of the vertebrae. The exposure provided by the pediculectomy is extended with resection of the transverse process and the medial portion of the rib and the rib head, along with resection of the entire costovertebral articulation (Fig. 4).

The patient is placed in a prone or lateral decubitus position. Three types of incisions can be made, depending on the lesion: paramedian, oblique, or T-shaped. The paramedian incision is made three fingerbreadths lateral to the spinous process. Oblique incisions follow the rib to be resected and extend across the midline. This is the preferred incision for localized lesions such as neurofibromas. A T-shaped incision allows exposure of the spinal column at multiple levels and resection of multiple ribs (Fig. 5). The transverse limb is made at the level of the 


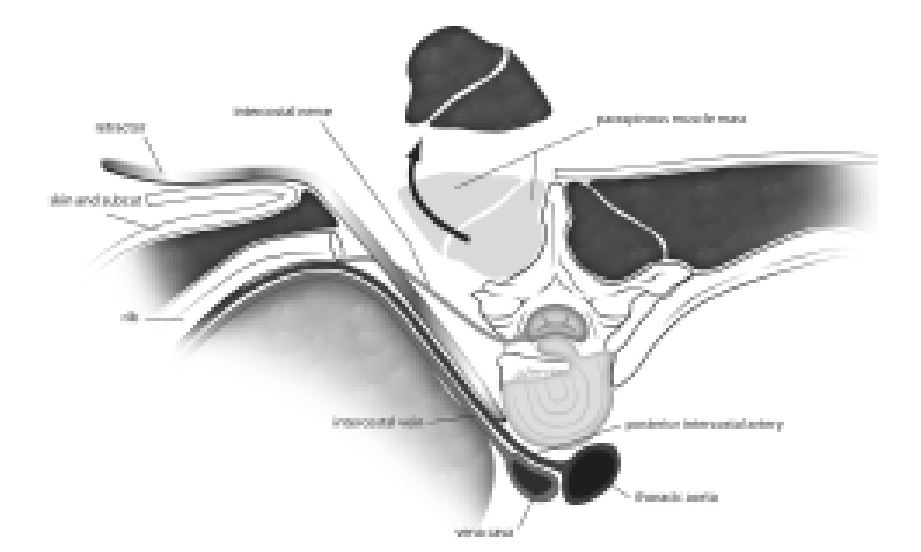

FIG. 4. Diagram of a costotransversectomy for treatment of a paraspinal NST. The transverse process, the medial portion of the rib, the rib head, and the costovertebral ligament are removed. subcut $=$ subcutaneous tissue.

vertebra to be exposed. This approach is favored for more extensive lesions and in cases in which fusion with instrumentation might be necessary.

Once the skin incision has been made, the trapezius and/or latissimus dorsi are divided along the skin incision to expose the rib. The intrinsic muscles of the back are separated from the spinous processes. The dissection is continued with a rasp from the vertebral arches and transverse processes above and below the transverse incision. The longissimus muscle is transversely dissected and retracted superiorly and inferiorly, exposing the ribs, laminae, facet joints, and transverse processes. The proximal 5 to $6 \mathrm{~cm}$ of the rib is resected.

The dissection of the rib is begun by exposing the lower border of the rib in a subperiosteal fashion from lateral to medial. The upper border is similarly dissected from a medial to lateral direction until the circumference of the rib is exposed. The subperiosteal dissection is carried to the costotransverse joint, which is then opened with a knife. The transverse process to the lamina is exposed subperiosteally. The transverse process may be removed at its base. The laterally separated rib is lifted and the periosteum below the rib is dissected to the costovertebral articulation. The neurovascular bundle lying caudal to the rib should be spared. After the periosteum is dissected, the rib can be removed.

Once the rib has been removed, the endothoracic fascia below the rib periosteum and the parietal pleural is retracted from the anterior side of the vertebrae and intervertebral discs. The neurovascular bundles should be spared. Flexible spatulas can be inserted to allow lateral visualization of two to three vertebrae. The sympathetic chain is identified and released with disconnection of the rami communicantes. The intercostal muscles are dissected off the segmental vessels. If necessary, the intercostal vessels may be ligated and transected anterior to the $\mathrm{VB}$, but the segmental nerves should be spared.

The spinal canal is exposed with an ipsilateral laminectomy, facetectomy, and pediculectomy. The lateral aspect of the dura mater and spinal cord can be seen. The exposed nerve roots are ligated and retracted, exposing the VB from the posterior to the anterior cortex. The tumor mass may be removed en bloc by turning it anteriorly, using the anterior margin of the vertebra as a hinge and avoiding injury to the spinal cord. If the parietal pleural has been injured during the procedure, a chest tube is necessary. Wound closure is accomplished by reapproximation of the divided muscle layer.

Approaches to Lumbar Tumors. Paraspinal NSTs of the lumbar spine can be approached through retroperitoneal approaches with a paramedian or flank incision.

Retroperitoneal Approach With a Paramedian Incision. This approach uses the retroperitoneal approach through a paramedian incision and is best employed for the L4-S1 interspace, although it may also be used for the L2-L4 levels.

The patient is plqced in the supine position, with the hip and knee joints slightly flexed and the hip joints abducted. A bump is placed under the low back at the level of the anterior superior iliac spine to form the lumbar lordotic curve. A curved incision is made just at the lateral aspect of the rectus abdominis sheath. The umbilicus is at the level of the L3-L4 intervertebral disc. The superior iliac crest is at the L4-L5 level. The L5-S1 level is located midway between the umbilicus and the pubic symphysis. The incision starts 4 to $5 \mathrm{~cm}$ superior to the umbilicus and extends to 5 to $6 \mathrm{~cm}$ superior to the pubic symphysis (Fig. 6). After the anterior rectus abdominis sheath is incised, the rectus muscle is mobilized and retracted medially to expose the posterior sheath. The inferior epigastric vessels lie between the abdominal wall muscles and the transversalis fascia. Due to their lateral position, these vessels are rarely at risk during a midline approach but may be injured during a more lateral approach. Otherwise the rectus muscle can be split and the posterior sheath approached between the muscle fibers. After the rectus muscle is swept away, the posterior rectus sheath is grasped and carefully incised so as not to cut through the peritoneum. The incision of the posterior sheath provides the entry into the retroperitoneal space.

Once the peritoneum is identified, it is swept off the abdominal wall with a blunt dissection. An alternative method is to incise the fibers of the transversalis fascia below the arcuate line. The blunt dissection allows the surgeon to find the plane between the peritoneum and the transversalis fascia, which can be incised rostrally. The retroperitoneal fat and intraabdominal contents are retracted medially, exposing the psoas muscle. The genitofemoral nerve is seen on the surface of the psoas muscle. The ureter can usually be identified to cross the common iliac artery from lateral to mediocaudal as the peritoneum is lifted away from the psoas. Great care needs to be taken to avoid injury to these structures.

The dissection is carried anterior and medial to the left common iliac vessels. The middle sacral artery and vein may be taken with ligation. The left iliac vein needs to be widely mobilized to allow placement of the retractor against the left lateral edge of the spine.

The location of the bifurcation of the abdominal aorta varies from the level of the upper L-4 VB to the upper L5 level. The location of the inferior vena cava confluence is slightly lower than the aortic bifurcation, ranging from the upper L-5 to the lower L-5 level. The L-5 approach is easy to perform in patients with a high bifurcation. The 

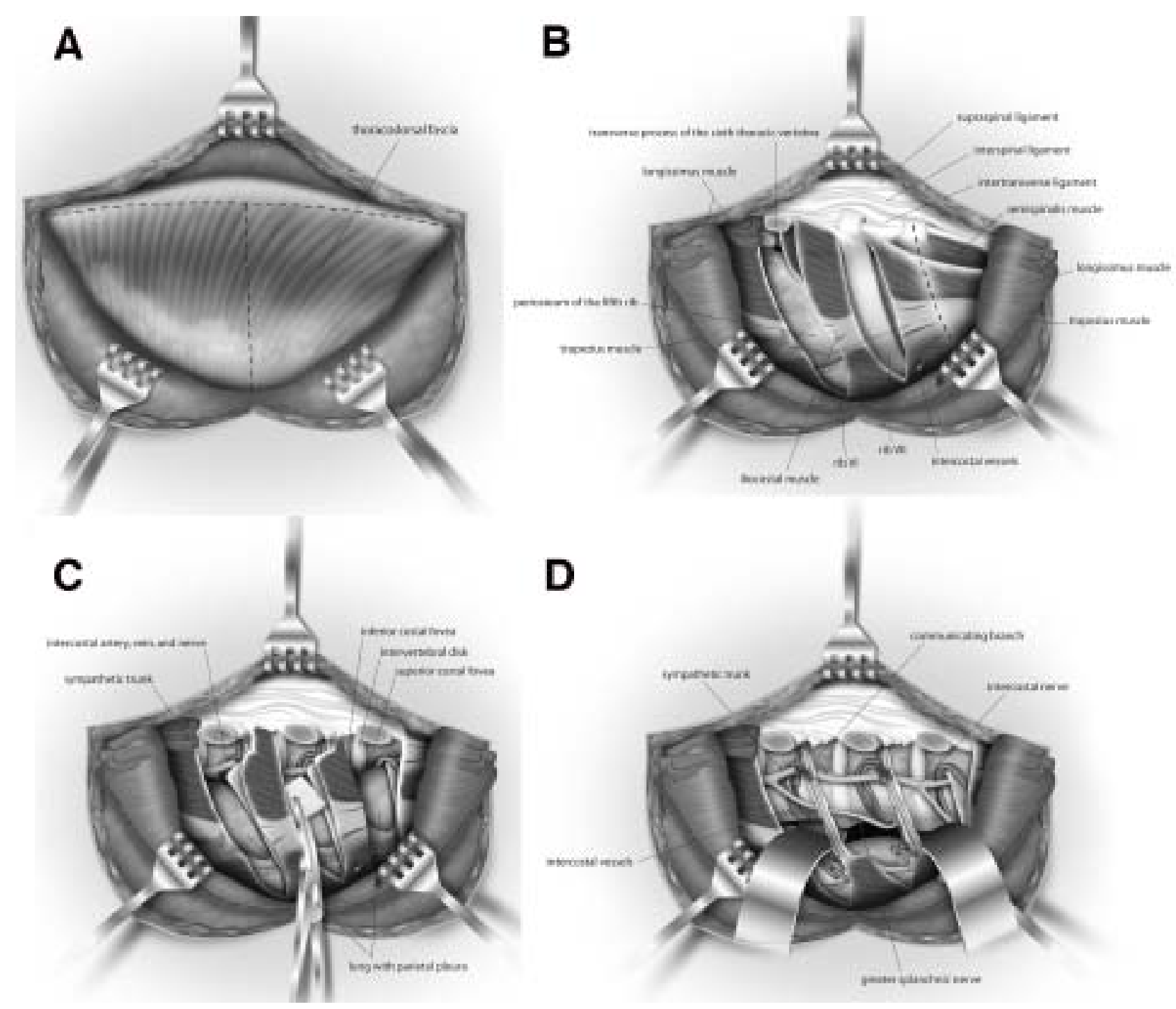

FIG. 5. Costotransversectomy for treatment of a paraspinal NST. Artist's illustration showing the T-shaped skin and muscle incision (A), the muscle division and rib resection (B), and exposure of the ventral surface of the VB (C and D).

bilateral retraction of common iliac vessels provides adequate exposure of the L-5 VB. For patients with bifurcation at the L4-5 disc level, an upward retraction of the bifurcation point of the great vessels is necessary. In patients with a low bifurcation, the retraction of the aorta and vena cava is done from the left to the right side to expose the L5 VB. For the vena cava to be retracted, the iliolumbar and fifth lumbar veins should be located and ligated. The iliolumbar vein has a tethering effect on the common iliac vein. For the L-5 segmental vessels and iliolumbar vein to be found, the medial margin of the psoas muscle should be retracted to the lateral side.

For exposure of the L-4 VB, the psoas muscle is retracted to expose the L-4 segmental vessels. These vessels are cut and dissected from the VB. The great vessels and the superior hypogastric plexus are retracted in the medial direction. For easy retraction of the great vessels, the L-5 segmental vessels and iliolumbar vein may be ligated.

Retroperitoneal Approach with Flank Incision. The lat- eral retroperitoneal approach to the L2-5 VBs gives access to the lumbar spine from the L-1 VB to the L4-5 disc space. Generally, the approach is made from the left side with the patient in the lateral decubitus position because the spleen is easier to retract than the liver, and the unique anatomy of the bifurcation of the great vessels makes the approach to the L4-5 disc space much easier from the left. The superior or ipsilateral leg should be flexed to relax the psoas muscle and to allow for gentler retraction of the ipsilateral lumbosacral plexus.

The skin incision should be centered between the lowest rib and the iliac crest, with minor adjustments made depending on the appropriate level. The incision should be S-shaped, with the abdominal aspect curving inferiorly as it approaches the midline and the posterior aspect curving gently cephalad. This is done to avoid cutting the rectus abdominis ventrally and the quadratus lumborum dorsally. The incision is extended down to the external oblique fascia. 

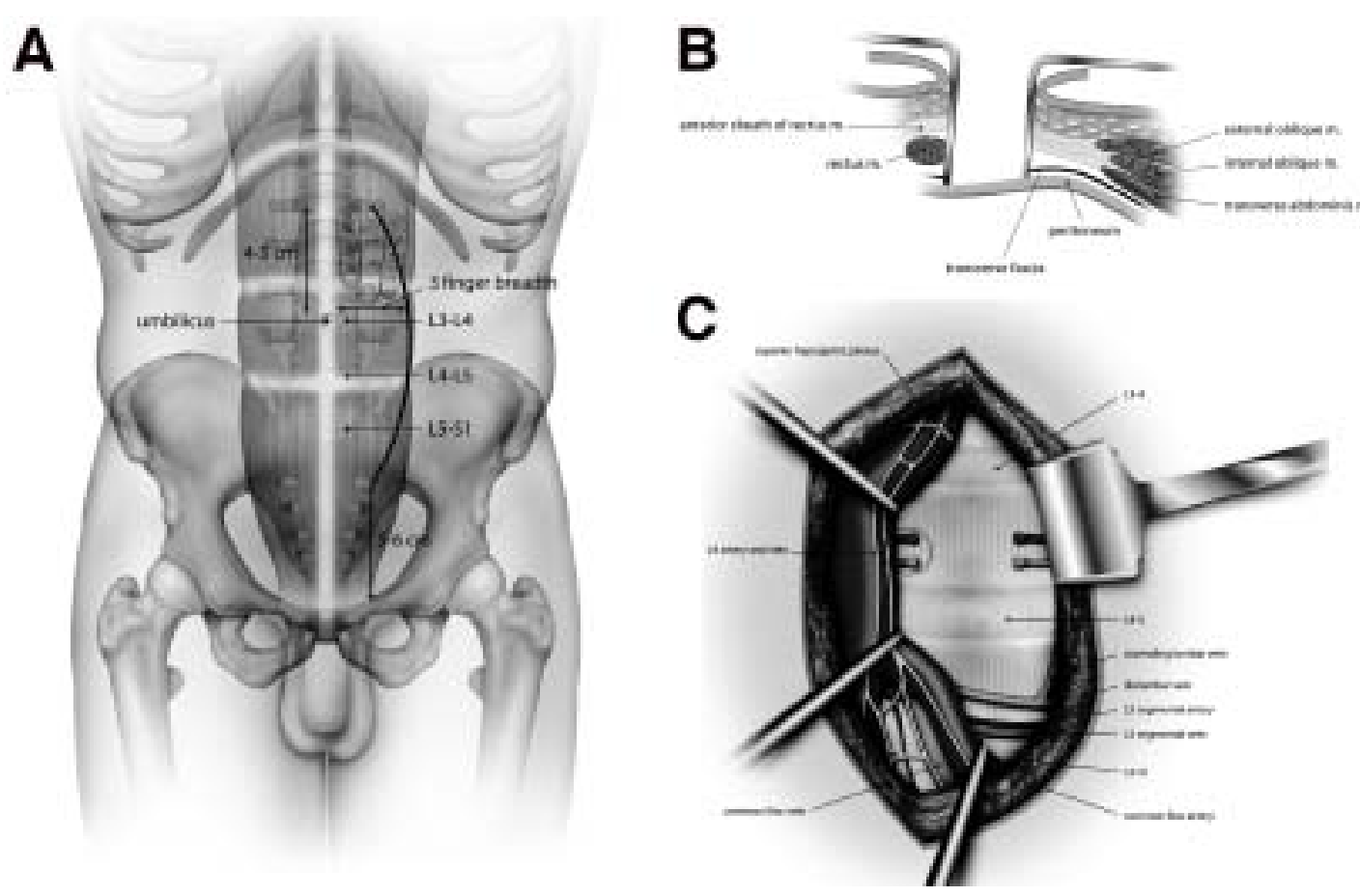

FIG. 6. Retroperitoneal approach with a paramedian incision to the lumbar paraspinal nerve sheath tumors. Artist's illustrations showing the skin incision (A), exposure of the peritoneum with retraction of the rectus abdominus sheath (B), and exposure of the L-4 VB (C) with retraction of the aorta to midline and after ligation and transection of the L-4 segmental vessels.

For more common muscle cutting, the external and internal obliques are approached, and the transversus abdominis is incised. The rectus abdominus should be spared at the medial aspect of the incision. Upon incising the transversus abdominis, the loose areolar tissue of the retroperitoneal space is visualized. The thin translucent peritoneum can be seen and the plane is developed with a blunt dissection. The peritoneum is gently swept off the posterior abdominal walls and the abdominal surface of the diaphragm. The abdominal contents that are being held by the peritoneum are then retracted medially. When mobilizing the kidney medially, one should retract the renal fascia and take care not to enter into the pararenal fat. Retroperitoneal procedures in general offer several potential advantages over transperitoneal approaches to the lumbar spine. Retroperitoneal approaches obviate the risk of small bowel obstruction or postoperative intraperitoneal adhesions. Because the autonomic plexus is not dissected, there is a reduced risk of retrograde ejaculation as compared with the transperitoneal approach. Additionally, the lateral decubitus position facilitates exposure of the lumbar spine, as gravity helps in pulling the abdominal contents anteriorly.

The psoas muscle is identified; this is the key to identifying the spine. The medial aspect of the psoas is attached to the spine, and the VBs and disc spaces can be palpated. The genitofemoral nerve is usually visualized on the surface of the psoas muscle. Once a disc space is identified, the psoas muscle is dissected off the VB by incising its attachments to the fibrous arcades that form its origin on the VB. Once the psoas has been retracted, the anterior spine is visualized, along with the segmental vessels and the sympathetic trunk. Less psoas dissection will be necessary in the lower lumbar spine.

Alternatively, the VB can be accessed through the psoas muscle belly. The dissection is carried out in a longitudinal fashion in line with the muscle fibers and through the anterior third of the psoas muscle. The intervertebral disc space often can be palpated through the psoas muscle and is exposed first. The respective middle portions of the adjacent VBs are then exposed. The segmental vessels are ligated and divided in the anterior half of the VB to allow the maximum possible collateral circulation to the neural foramen and spinal cord.

\section{Results}

A total of 88 paraspinal NSTs were treated surgically over the 36-year study period: 56 schwannomas (63.6\%), seven solitary neurofibromas $(8.0 \%), 21 \mathrm{NF} 1$-associated neurofibromas (23.9\%), and four malignant parspinal NSTs $(4.5 \%)$ (Table 1). Schwannomas accounted for the majority of paraspinal NSTs. The paraspinal NSTs were in the cervical spine in $53.4 \%$ of cases, the thoracic spine in $23.9 \%$, and the lumbar spine in $22.7 \%$.

Schwannomas were found in the cervical area in 28 cases $(50.0 \%)$, the thoracic in $16(28.6 \%)$, and lumbar in $12(21.4 \%)$. The NF1-associated neurofibromas were mostly confined to the cervical area in 14 patients $(66.7 \%)$, but also occurred in the lumbar in four $(19.0 \%)$ and thoracic in three (14.3\%). Solitary neurofibromas were uncommon and showed a slight preponderance for the cervical area (three cases, $42.8 \%$ ), occurring in the thoracic and lumbar spine in two cases $(28.6 \%)$ each. 
TABLE 1

Paraspinal NSTs surgically treated at Louisiana State University Health Sciences Center from 1970 to 2006

\begin{tabular}{lcllll}
\hline \hline & \multicolumn{5}{c}{ No. of Lesions (\%) } \\
\cline { 2 - 6 } \multicolumn{1}{c}{ Spinal } \\
\cline { 2 - 6 } Level & Schwannoma & Solitary & NF1-Associated & Malignant & All Para- \\
Paraspinal NSTs & Neurofibromas & spinal NSTs \\
\hline cervical & 28 & 3 & 14 & 2 & $47(53.4)$ \\
thoracic & 16 & 2 & 3 & 0 & $21(23.9)$ \\
lumbar & 12 & 2 & 4 & 2 & $20(22.7)$ \\
all levels & $56(63.6)$ & $7(8.0)$ & $21(23.9)$ & $4(4.5)$ & $88(100.0)$ \\
\hline
\end{tabular}

Malignant paraspinal NSTs were rare, occurring exclusively in the cervical and lumbar spines.

Pain and paresthesia were the most common presenting symptoms, occurring in $79(90 \%)$ and 81 (92\%) of patients, respectively. Weakness was the presenting complaint in 28 (32\%) of patients. Twelve patients (13.6\%) presented with myelopathy. Duration of follow-up ranged from 6 to 120 months. The average follow-up period was 18 months.

A total resection of the tumor was achieved in 50 (89.3\%) of the patients with schwannomas and 22 $(78.6 \%)$ of the patients with neurofibromas. There was a large reduction of pain in $70(88.6 \%)$ of the 79 patients who had preoperative pain, and weakness improved in 18 $(64.3 \%)$ of 28 patients. Postoperative weakness occurred in $12(42.9 \%)$ of these patients, although the symptom improved in most $(83.3 \%)$ of these patients over a 12month period. The majority of them had neurofibromas. Myelopathy similarly improved in eight (66.7\%) of 12 patients. Only two patients with malignant paraspinal NSTs were alive 46 months postoperatively.

\section{Discussion}

Paraspinal NSTs account for one fourth to one third of spinal neoplasms. ${ }^{6,11}$ Only about 8 to $10 \%$ show extraforaminal and intraforaminal involvement. ${ }^{3}$ Paraspinal nerve schwannomas are generally reported to be evenly distributed throughout the spinal canal, though some authors have noted increased prevalence in the cervical and lumbar spine. ${ }^{11}$ Approximately $10 \%$ of posterior mediastinal tumors are paraspinal nerve sheath tumors. ${ }^{7,13,14}$ Isolated neurofibromas are less frequent and occur mainly in patients with NF1. The majority of paraspinal NSTs are benign. Malignant transformation is rare and usually occurs in the setting of plexiform neurofibromas in NF1 patients. Unfortunately malignant paraspinal NSTs carry a very poor prognosis.

Initial symptoms depend on the level of the tumor and the extent of motor deficit and spinal cord compression. Patients with high cervical lesions are more likely to present with sensory disturbances until the tumor is large enough to compress the spinal cord. For this reason, largediameter intraspinal tumors in the high cervical spine tend to be identified later than those in other areas. In cases of lumbar paraspinal tumors, weakness in the lower extremity may also not occur until later in the disease process, when the tumors are large enough to cause compression of the cauda equina. In cases of tumors of the thoracic spine, pain is the usual presenting sign, although spinal cord compression can result in myelopathy. ${ }^{5}$

The extent of postoperative improvement of symptoms depends on several factors. Patients who have severe deficits preoperatively are less likely to experience improvement. In our series, $66.7 \%$ of patients with myelopathy experienced improvement, compared with $85.7 \%$ of patients who presented with pain and paresthesia. These findings are consistent with the experience of other authors. Patients with pain and paresthesia usually have pain relief after surgery. Some patients will experience a sensory deficit. In a minority of patients, pain may worsen. This variety of response is due to the widespread nature of the disease and fascicular involvement of neurofibromas. In addition, neurofibromas can recur in patients with NF1. Klekamp and Samii ${ }^{6}$ evaluated patients with NSTs and NF2 and found that individuals with NF2 were much less likely to experience improvement in symptoms. The recurrence rate of tumors in patients with NF2 is higher and repeated operations are complex.

It is difficult to differentiate schwannomas from neurofibromas on the basis of imaging characteristics. Schwannomas are generally intradural and extramedullary. Only $15 \%$ are extradural or dumbbell shaped. They are generally small, but larger paraspinal extensions do occur. Most are in the thoracic region; they are equally distributed in the cervical and lumbar regions. ${ }^{8}$

Neurofibromas can be intradural extramedullary or paraspinal and they can have different degrees of involvement of the spinal root, plexus, peripheral nerves, and end organs. They are usually associated with NF1 and tend to occur at the cervical level more often than the thoracic and lumbar levels. Their sizes vary, but these lesions are generally larger in the lumbar spine. Three types of neurofibromas are identified: localized, diffuse, and plexiform.

Imaging characteristics are similar for schwannomas and neurofibromas. ${ }^{4,8}$ Bony findings may include a widened interpedicular distance, VB erosion, or enlarged neural foramina. Myelography may reveal a block in cerebral spinal fluid flow. ${ }^{8,11}$ Computed tomography often reveals well-marginated masses and erosion and remodeling of adjacent bone. Cystic changes can appear, but rarely hemorrhage or calcifications. The best imaging modality for evaluation of these lesions is MR imaging. On unenhanced T1-weighted images, schwannomas usually appear isointense or hypointense relative to the spinal cord and nerve roots. On T2-weighted images, NSTs are usually hyperintense. Schwannomas, especially large ones, may show characteristic cystic changes with heterogeneity. 
Less frequently, hemorrhage will be apparent. The classic "target sign," an enhancing rim with a central area of hypointensity, will occasionally be present. In T1-weighted images obtained after administration of contrast medium, the lesions show intense enhancement which may be uniform or heterogeneous, depending on the level of cystic changes. ${ }^{8}$

In addition, the split-fat sign, a rim of fat surrounding the tumor, may be present in schwannomas or neurofibromas. ${ }^{8} \mathrm{~A}$ low-intensity tumor capsule may be seen in $70 \%$ of schwannomas and 30\% of neurofibromas. Eccentrically placed lesions on a nerve may help differentiate a schwannoma from a neurofibroma. The imaging characteristics of the lesions are similar in NF1 and NF2.

Most malignant paraspinal NSTs arise from plexiform neurofibromas. Approximately 5\% of patients with NF1 develop malignant paraspinal NSTs. They share imaging characteristics of other neurogenic tumors. Computed tomography and MR imaging cannot be relied on to differentiate between benign and malignant lesions. There are, however, several characteristics that should raise the level of suspicion for malignancy: lesion size larger than $5 \mathrm{~cm}$, enlarging tumors, ill-defined margins, invasion of fat planes, heterogeneity with central necrosis, and peritumoral edema. ${ }^{8}$

Paraspinal NSTs represent unique surgical challenges. The surgical approach depends on the spinal level of the tumor, and these lesions can span several levels. Resection of NSTs involves a strong appreciation of the threedimensional anatomy of the spine and surrounding structures. When treating cervical lesions, care must be taken to avoid injury to the VA, which is usually displaced or embedded in the tumor. In cases of intraspinal involvement, care must be taken to avoid injury to the spinal cord. Multilevel resections may result in spinal instability and may necessitate spinal stabilization with instrumentation. The thoracic spine must always be approached with great care. Although nerve roots can be sacrificed, surgical approaches to the upper and middle thoracic spine must avoid injury to the spinal cord and great vessels. Surgical exposure at these levels is often limited. In the lower thoracic and lumbar spine, exposure is further complicated by the need to bring down the diaphragm and avoid injury to the nerves running in the retroperitoneal space and the psoas muscle. Traditional approaches to the thoracic and lumbar spine result in long operative times and high morbidity for the patient. Less invasive approaches and an understanding of the complex anatomy behind these exposures are necessary.

Surgery for the treatment of any paraspinal NST usually involves complete resection of the nerve roots involved. Even a large asymptomatic paraspinal tumor can be removed totally with an appropriate surgical approach and intraoperative electrophysiological recording. When eloquent nerve roots are sacrificed, it is important to evaluate the extent of neurological recovery that can be expected to occur. The majority of patients who undergo surgery for paraspinal NSTs will present with pain and paresthesia. Often, however, patients will present with weakness or myelopathy. Celli ${ }^{2}$ reported on 26 patients who underwent resection of 27 schwannomas or neurofibromas. Only $14.8 \%$ of them experienced worsening radicular function postoperatively. Permanent disability occurred in $7.4 \%$ of cases. Other authors have found similar results. Resection of the involved nerve root is not likely to lead to permanent deficit, and most patients recover function over a 6to 12-month period. ${ }^{9}$ Neurofibroma resections tend to result in a higher rate of permanent disability than schwannoma resections. Patients with neurofibromatosis are less likely to improve to the same extent as patients with schwannomas, given the expansive nature of the disease and the widespread involvement. ${ }^{10}$ Neural sheath tumors grow slowly. It is likely that function of the relevant nerve root has already been compensated for by other adjacent nerve roots. In addition, large tumors may compress adjacent nerve roots, and this compression may be relieved by surgical resection. Some authors advocate the use of preoperative electromyography/nerve conduction velocity studies to assess nerve root function. At our institution, intraoperative electrophysiological recording was performed in all patients who underwent surgery for treatment of paraspinal NSTs. Intraoperative evidence of loss of function of the involved nerve root seemed to indicate a lower probability of postoperative deficit. In our study, the majority of patients who presented with a preoperative and postoperative deficit improved. As in other studies, patients with neurofibromatosis did not do as well as other patients.

\section{Conclusions}

Paraspinal NSTs may occur along the entire neuroaxis and present unique surgical challenges, given their anatomical relationships to the spine, spinal cord, nerve roots, and major vasculature. The surgical approach for treatment of a paraspinal NST depends on the location of the lesion and its relationship to the paraspinal anatomy. Surgery is indicated for patients with intractable pain and paresthesia, weakness, myelopathy, or spinal cord compromise. Patients with severe pain and paresthesia may benefit from resection and may experience significant improvement in symptoms. Patients with NF1 and NF2 require special consideration and may not show the same level of clinical improvement.

\section{References}

1. Akeyson EW, McCutcheon IE: Single-stage posterior vertebrectomy and replacement combined with posterior instrumentation for spinal metastasis. J Neurosurg 85:211-220, 1996

2. Celli P: Treatment of relevant nerve roots involved in nerve sheath tumors: removal or preservation? Neurosurgery 51: 684-692, 2002

3. Conti P, Pansini G, Mouchaty H, Capuano C, Conti R: Spinal neurinomas: retrospective analysis and long-term outcome of 179 consecutively operated cases and review of the literature. Surg Neurol 61:34-44, 2004

4. Egelhoff JC, Bates DJ, Ross JS, Rothner AD, Cohen BH: Spinal MR findings in neurofibromatosis types 1 and 2. AJNR Am J Neuroradiol 13:1071-1077, 1992

5. Jinnai T, Koyama T: Clinical characteristics of spinal nerve sheath tumors: analysis of 149 cases. Neurosurgery 56: 510-515, 2005

6. Klekamp J, Samii M: Surgery of spinal nerve sheath tumors with special reference to neurofibromatosis. Neurosurgery 42:279-290, 1998 
7. Konno S, Yabuki S, Kinoshita T, Kikuchi S: Combined laminectomy and thoracoscopic resection of dumbbell-type thoracic cord tumor. Spine 26:E130-E134, 2001

8. Pilavaki M, Chourmouzi D, Kiziridou A, Skordalaki A, Zarampoukas T, Drevelengas A: Imaging of peripheral nerve sheath tumors with pathologic correlation: pictorial review. Eur J Radiol 52:229-239, 2004

9. Schultheiss R, Gullotta G: Resection of relevant nerve roots in surgery of spinal neurinomas without persisting neurological deficit. Acta Neurochir (Wien) 122:91-96, 1993

10. Seppala MT, Haltia MJ, Sankila RJ, Jaaskelainen JE, Heiskanen O: Long-term outcome after removal of spinal neurofibroma. J Neurosurg 82:572-577, 1995

11. Seppala MT, Haltia MJ, Sankila RJ, Jaaskelainen JE, Heiskanen O: Long-term outcome after removal of spinal schwannoma: a clinicopathological study of 187 cases. J Neurosurg 83: 621-626, 1995
12. Takase K, Sawamura Y, Igarashi K, Chiba Y, Haga K, Saito H, et al: Demonstration of the artery of Adamkiewicz at multidetector row helical CT. Radiology 223:39-45, 2002

13. Takeda S, Miyoshi S, Minami M, Matsuda H: Intrathoracic neurogenic tumors-50 years' experience in a Japanese institution. Eur J Cardiothorac Surg 26:807-812, 2004

14. Vallieres E, Findlay JM, Fraser RE: Combined microneurosurgical and thoracoscopic removal of neurogenic dumbbell tumors. Ann Thorac Surg 59:469-472, 1995

Manuscript submitted March 23, 2007.

Accepted May 8, 2007.

Address reprint requests to: Daniel H. Kim, M.D., Department of Neurosurgery, Ochsner Clinic Foundation, 1514 Jefferson Highway, New Orleans, Louisiana 70121. email:neurokimdaniel@ yahoo.com. 